\title{
HIGH FIDELITY IMAGING ALGORITHM FOR THE UNDIQUE IMAGING MONTE CARLO SIMULATOR
}

\author{
Grégoire Tremblay ${ }^{1 *}$, Gilles Roy ${ }^{2}$ \\ ${ }^{I}$ AEREX Avionics, 324 St-Augustinav.,Lévis, Qc, Canada, G6Z 8G8,gtremblay@aerex.ca \\ ${ }^{2}$ DRDC Valcatier, 2459 de la Bravoure, Québec, Qc, Canada, G3J $1 X 5$
}

\begin{abstract}
The Undique imaging Monte Carlo simulator (Undique hereafter) was developed to reproduce the behavior of 3D imaging devices. This paper describes its high fidelity imaging algorithm.
\end{abstract}

\section{INTRODUCTION}

The field of 3D imaging is evolving rapidly. Advance in Flash ladar [1-2], simplified the task of laser 3D imaging. These technologies can be used for observation of targets through foliage [1], aerosol [3], or netting [2].

We developed Undique in an effort to understand the influence of these phenomena on Flash lidar image quality. The simulator reproduces not only the scattering of the photon in the propagation range but also $3 \mathrm{D}$ solid targets and basic receiver behavior.

In section 2 we give a brief description of Undique capabilities. Section 3 describes the high fidelity imaging algorithm. Finally, section 4 presents some examples of simulated results.

\section{UNDIQUE}

Undique (un-di-kwe) is a latin word meaning "from all parts", a name quit adequate when propagating photons in aerosols. It is a versatile tool developed to reproduce the behaviour of different types of active imaging system such as classical lidar system, passive 2D imager, active imager, or range gated 3D imager. To simulate these systems, we need a 2D gated detection system, a source, and a propagation range. In the case of 3D range gated imager, a solid 3D target is also simulated. Fig. 1 shows a schematic view of the multiple components emulated by the system.

\subsection{Simulated sub systems characteristics}

Undique generates individual photons. The source is monochromatic but its wavelength can be adjusted before simulations. The photons polarization state is defined using the Stokes parameters. To simulate a gated camera, we keep a record of the total distance travelled by the photons.

The source can be located anywhere in the simulated environment. Multiple sources or predefined source patterns such as bar patterns of radial patterns can be used.

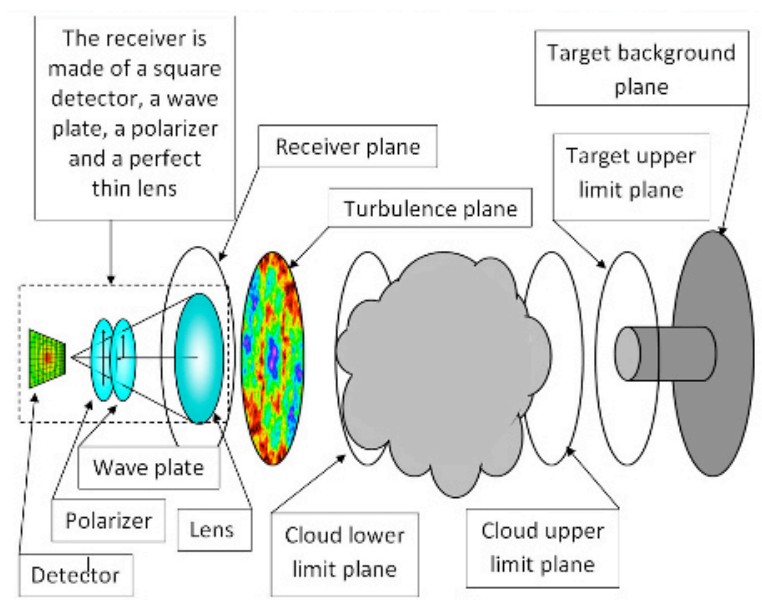

Fig. 1. The Undique Monte Carlo simulation environment. We show the various components of the system.

The gated camera is made of a perfect thin lens and a square detector. The aperture diameter, the focal length and the focus of the camera can be changed. To provide polarization analysis capability, a polarizer and a retarder are located in front of the detector.

The propagation range is the distance between the camera and the solid target. It is divided in a series of propagation planes. Each plane generates a specific behavior. The simulation basic planes are the camera's aperture plane, the solid target upper limit, and the target background plane. We also have planes to define the obscurant limits and for turbulence effects (still under development). Photons propagate from one plane to the next. When it arrives at a plane, actions are taken and propagation is modified accordingly. 
The solid target is at the end of the propagation range. It is a $3 \mathrm{D}$ structure that rests on an infinite solid background. We can have up to 20 individual targets. These targets can be cylindrical, rectangular, or have specific regular patterns. Albedo and BRDF of solid targets can be changed.

Undique relies on phase function information to scatter the photons. The first aerosols included in the simulator were spherical water droplets described by the Mie theory. The aerosols can be monodispersed or have a normal, log normal, bilog normal, or gamma distribution.

The simulator can also use Rayleigh scattering, Henyey Greenstein phase function and user defined phase function for atmospheric propagation. In water, Fournier Forand and pure water phase functions are available.

Aerosols can be located anywhere in the propagation range. It can span from the camera to the target or be concentrated in a determined position. We can simulate up to three clouds having either uniform, linearly changing, or parabolic extinction profile.

\subsection{Direct propagation and variance reduction}

When used in direct propagation mode, the simulator propagates ballistic photons until they are captured by the camera or reach a predetermined delay. In order to increase the throughput of the simulator, the variance reduction mode generates events every time an interaction occurs within the system Field of View (FOV). Three different variance reduction methods are used. The first method is the wellknown semi-analytical method [4] also known as the expected value method [5]. The second is an importance sampling method used to increase the amount of events occurring in front of the receiver. The third method is splitting and Russian roulette used to produce a defocusing effect.

\section{HIGH-FIDELITY IMAGE GENERATION}

Conventional atmospheric Monte Carlo [6] are usually coaxial with the source, focused at infinity, and observe symmetrical system. The camera aperture is not considered in the imaging process. The errors caused by these approximations are usually less important than the intrinsic variability of the simulation.
In recent years, active systems have been integrated in various industrial processes [7]. The aperture diameter can represent a few percent of the total range. In these conditions, the photon distribution over the camera's aperture can have a significant impact. When simulating engulfed system, the aperture's contribution is paramount.

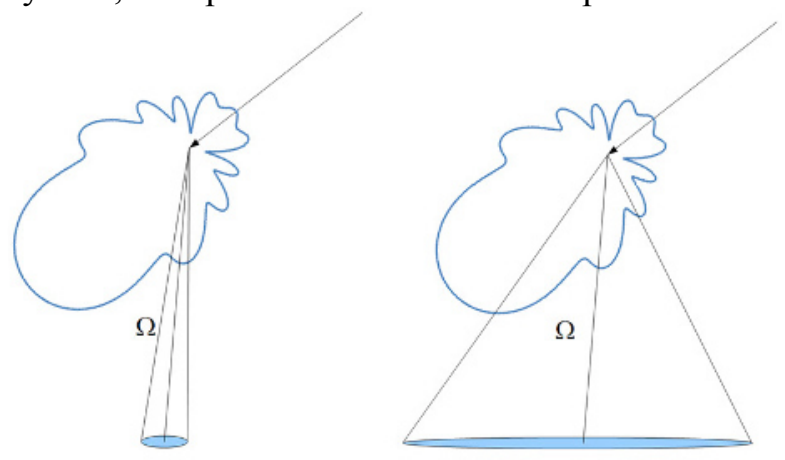

Fig. 2. Phase function projection over a small (left) and a large (right) solid angle $\Omega$.

In direct propagation mode, ballistic photons touching the camera aperture are focused on the detector respecting the optical properties of thin lenses. In variance reduction mode, a sub photon having the probability to reach the receiver is send toward it. This probability is provided by the phase function. Fig.2 shows the two possible cases. On the left, the range is large and the camera's aperture sustains a small solid angle $\Omega$. On the right the solid angle sustained by the aperture is large and the projection of the phase function changes considerably over it. When the camera's aperture is small compared to the distance to travel, the probability to go toward the small solid angle $\Omega$ is

$$
P_{\Omega}=\frac{F(\theta, \varphi)}{d^{2}} \pi r_{\text {aperture }}^{2},
$$

where $F(\theta, \varphi)$ is the phase function as a function of elevation $\theta$ and azimuth $\varphi, d$ is the range and $r$ the aperture radius. When the camera's aperture is larger, the phase function projection over the aperture varies. The scattered light can go toward a larger $\Omega$ and the new probability is

$$
P_{\Omega}=\frac{1}{d^{2}} \int_{\varphi=\phi_{0}}^{\varphi_{1}} \int_{\theta=\theta_{0}}^{\theta_{1}} F(\theta, \varphi) \sin \theta d \theta d \varphi .
$$

This calculation can be perform faster by putting $F(\theta, \varphi) \sin \theta d \theta$ in a look-up-table (LUT). 
We can either produce an exact mapping of the aperture's probability distribution to locate the photon or use an alternate, less computer time consuming strategy. We chose the latter and we explain how we proceeded in the next section.

\subsection{The photon pointing into the receiver}

In Fig. 3, an interaction occurs at point A inside the camera FOV, and the photon travels along line $\mathrm{AB}$; point $\mathrm{B}$ is inside the aperture. The circumference of the aperture is divided in 24 sections of $15^{\circ}$. They are used to define 24 slices having point $\mathrm{B}$ as vertex. Knowing the interaction coordinate in $\mathrm{A}$, the contact point coordinate in $\mathrm{B}$, and the edge coordinate of a slice in $\mathrm{E}$, we can find the elevation angle $\theta$ defined by these three points. Knowing the azimuthal angle $d \varphi$ sustained by the slice, we can find the probability to be scattered in that slice using Equ. 2. The operation is repeated for all the slices and we get a probability distribution. A random number is drawn to determine the slice touched by the photon. Using the LUT as a probability distribution along elevation, a second random number is used to find the photon location in $\theta$ and a third is used to determine $\varphi$.

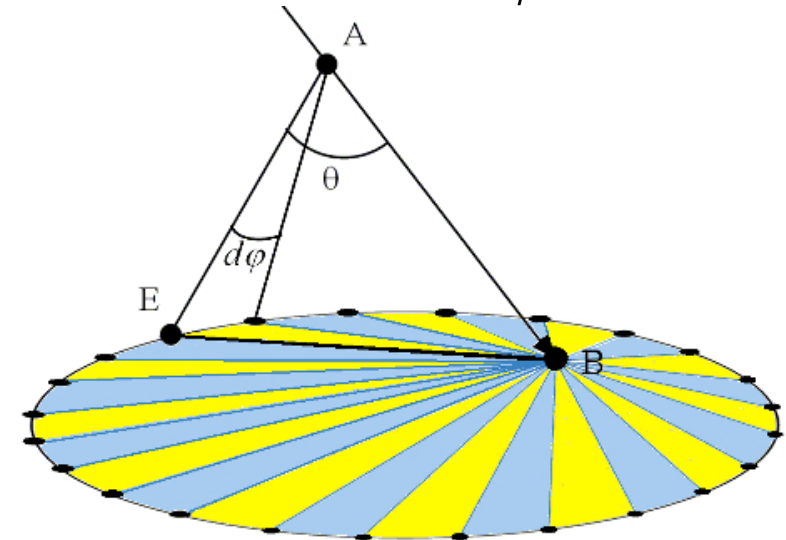

Fig. 3. Schematic view of the photon distribution on the camera's aperture in variance reduction: the photon pointing inside case. The slices are shown in yellow and blue.

\subsection{The photon pointing outside the receiver}

This situation is shown in Fig. 4. The interaction between the photon and the aerosol happens at point $\mathrm{A}$ but this time the projected location of the photon is outside the aperture (point B). The aperture is divided in 24 slices having the same angular width $d \varphi$. A line is traced between $\mathrm{B}$ and
E, the far edge of the aperture. The probability to be scattered between point $\mathrm{D}$ and $\mathrm{E}$ is computed for every slice using $\theta_{1}, \theta_{2}$, and $d \varphi$ in Equ. 2 . From that point, the distribution of the photons on the aperture is similar to the previous case.

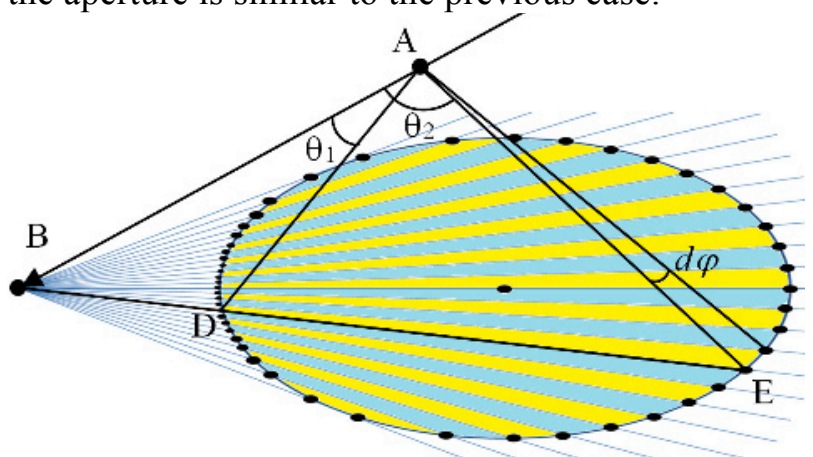

Fig. 4. Schematic view of the photon distribution on the camera's aperture in variance reduction: the photon pointing outside the aperture case. The slices are shown in yellow and blue.

\section{SOME EXAMPLES}

In this section we present simulations made using Undique to demonstrate its versatility. The first example in Fig. 5 represents a solid target made of concentric cylinders seen through aerosol. This was done to assess the impact of aerosol on our capacity to detect 3D shapes through obscuration [8].

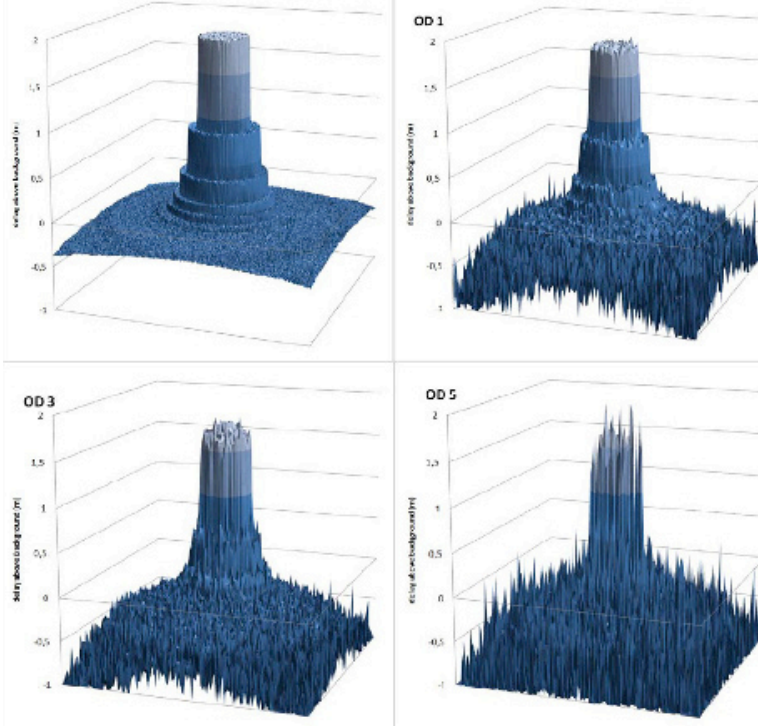

Fig. 5. Simulation of a solid target seen through an aerosol made of $10 \mu \mathrm{m}$ water droplets. Top left: OD0. Top right: OD1. Bottom left: OD3. Bottom right: OD5 (from [8]). 
In Fig. 6 we look at a radial source through an $\mathrm{F} / 2$ system. The source-camera distance is equal to 100 times the aperture diameter and the FOV is $50 \mathrm{mrad}$. On the left, the source is focused; on the right, defocus generates the expected phase inversion in the radial pattern. This is only possible if the photons are distributed adequately on the aperture.

In Fig. 7 we show the case of a polarised signal propagating through a test case. The case is chosen because it can be calculated analytically and because it is highly sensitive to small errors in polarization management.

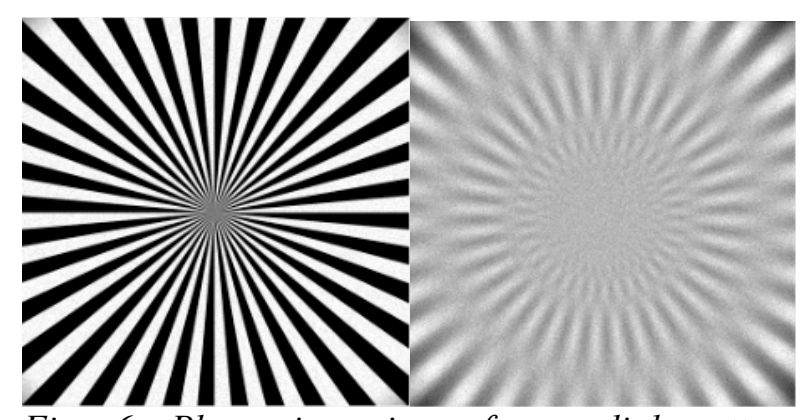

Fig. 6. Phase inversion of a radial pattern generated by defocus of the optical system.

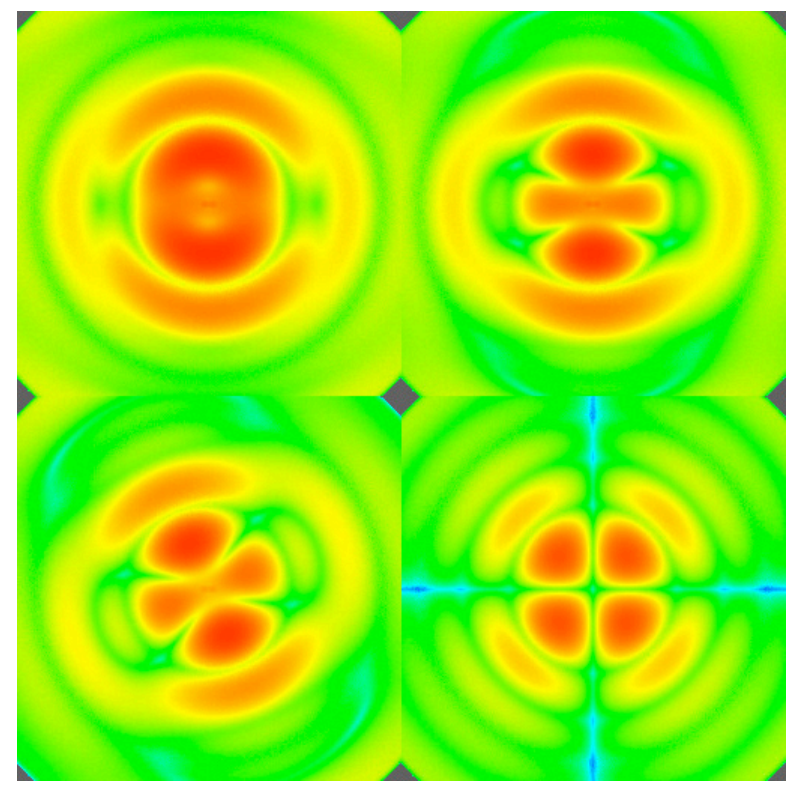

Fig. 7. Polarized source reflected by aerosols in a test case. We see the total reflected signal (top left), the principal polarization(top right), the $45^{\circ}$ polarization (bottom left) and the secondary polarization (bottom right).

\section{CONCLUSION}

The Undique Monte Carlo simulator is a versatile tool that can be used to reproduce most passive and active imager observing a scene under obscuration. Its unique imaging algorithm enables it to reproduce the behavior of actual systems under a wide range of conditions.

\section{REFERENCES}

[1] Murray, J.T., Moran, S.E., Roddier, N., Vercillo, R., Bridges, R., Austin, W., "Advanced 3D polarimetric flash ladar imaging through foliage", Proc. SPIE, 5086, 84-95 (2003).

[2] Halmos, M.J., "Eyesafe 3D flash Ladar for target under obscuration", Proc. SPIE, 5086, 7083 (2003).

[3] Trickey, E., Church, P., Cao, X., "Characterization of the OPAL Obscurant Penetrating LiDAR in various Degraded Visual Environments", Proc. SPIE, 8737, 87370E (2013).

[4] Poole, L.R., Venable, D.D., Cambell, J.W., Semianalytic Monte carlo radiative transfer model for oceanographic lidar systems, Appl. Opt. 20, 3653-3656 (1981).

[5] Spanier, J., Gelbard, E.M., "Monte Carlo Principles and Neutron Transport Problems", Dover (1969).

[6] Marchuk, G.I., Mikhailov, G.A., Nazaraliev, M.A., Darbinjan, R.A., Kargin, B.A., Elepov, B.S., The Monte Carlo Methods in Atmospheric Optics, Springer series in Opical Science, (1980).

[7] Berrocal, E., Meglinski, I., Jermy, M., "New model for light propagation in highly inhomogeneous polydisperse turbid media with applications in spray diagnostics", Opt. Express, 13, 9181-9195 (2005).

[8] Tremblay, g., Cao, X., Roy, G., "The effect of dense aerosol cloud on the 3D information content of flash Lidar", Proc. SPIE, 7828, 78280C (2010). 